\title{
The Relation between Perception and Brain Activity in Gaze-Evoked Tinnitus
}

\author{
Margriet J. van Gendt, ${ }^{1 \star}$ Kris Boyen, ${ }^{1,2 \star}$ Emile de Kleine, ${ }^{1,2}$ Dave R.M. Langers, ${ }^{1,2}$ and Pim van Dijk ${ }^{1,2}$ \\ ${ }^{1}$ Department of Otorhinolaryngology/Head and Neck Surgery, University of Groningen, University Medical Center Groningen, 9700 RB Groningen, The \\ Netherlands and ${ }^{2}$ Graduate School of Medical Sciences, Research School of Behavioural and Cognitive Neurosciences, University of Groningen, 9700 RB \\ Groningen, The Netherlands.
}

Tinnitus is a phantom sound percept that can be severely disabling. Its pathophysiology is poorly understood, partly due to the inability to objectively measure neural correlates of tinnitus. Gaze-evoked tinnitus (GET) is a rare form of tinnitus that may arise after vestibular schwannoma removal. Subjects typically describe tinnitus in the deaf ear on the side of the surgery that can be modulated by peripheral eye gaze. This phenomenon offers a unique opportunity to study the relation between tinnitus and brain activity. We used functional magnetic resonance imaging in humans to show that in normal-hearing control subjects, peripheral gaze results in inhibition of the auditory cortex, but no detectable response in the medial geniculate body (MGB) and inferior colliculus (IC). In patients with GET, peripheral gaze (1) reduced the cortical inhibition, (2) inhibited the MGB, and (3) activated the IC. Furthermore, increased tinnitus loudness is represented by increased activity in the cochlear nucleus $(\mathrm{CN})$ and IC and reduced inhibition in the auditory cortex (AC). The increase of $\mathrm{CN}$ and IC activity with peripheral gaze is consistent with models of plastic reorganization in the brainstem following vestibular schwannoma removal. The activity decrease in the MGB and the reduced inhibition of the AC support a model that attributes tinnitus to a dysrhythmia of the thalamocortical loop, leading to hypometabolic theta activity in the MGB. Our data offer the first support of this loop hypothesis of tinnitus, independent of the initial experiments that led to its formulation.

\section{Introduction}

Subjective tinnitus is a common hearing disorder with a potentially devastating impact on the quality of life, characterized by sound perception in the absence of an acoustic stimulus (Lockwood et al., 2002; Langguth et al., 2007; Roberts et al., 2010). It is typically associated with peripheral hearing loss. The physiology of tinnitus may involve increased spontaneous activity, increased bursting activity, or enhanced neural synchrony in the auditory system (Eggermont, 2000; Salvi et al., 2000; Kaltenbach, 2011). Existing studies suggest that tinnitus arises from plastic reorganization that involves a disruption of the normal balance between excitation and inhibition in the brain.

Somatic tinnitus is a special form of tinnitus in which the perceived sound can be elicited or modulated by bodily maneuvers (Levine et al., 2007). Prevalence estimates range from 20 to 78\% among tinnitus patients (Levine, 1999; Simmons et al.,

\footnotetext{
Received June 12, 2012; revised Aug. 31, 2012; accepted Sept. 21, 2012.

Author contributions: P.v.D. designed research; M.J.v.G. performed research; E.d.K. and D.R.M.L. contributed unpublished reagents/analytic tools; M.J.v.G. and K.B. analyzed data; K.B. wrote the paper.

This research was supported by the American Tinnitus Association, the Netherlands Organization for Scientific Research, and the Heinsius Houbolt Foundation. This study is part of the research program of our department, Healthy Aging and Communication. We thank Dr. Bernard van der Laan for providing clarification with regard to the surgery that the patients underwent.

*M.J.v.G. and K.B. contributed equally to this work.

The authors declare no competing financial interests.

Correspondence should be addressed to Kris Boyen, Department of Otorhinolaryngology/Head and Neck Surgery, University of Groningen, University Medical Center Groningen, The Netherlands, P.0. Box 30.001, 9700 RB Groningen, The Netherlands. E-mail: k.boyen@umcg.nl.

DOI:10.1523/JNEUROSCI.2791-12.2012

Copyright $\odot 2012$ the authors $\quad 0270-6474 / 12 / 3217528-12 \$ 15.00 / 0$
}

2008). One rare example is gaze-evoked tinnitus (GET) in which the tinnitus is modulated or elicited by eye movements. GET has been mainly described after surgical extirpation of a vestibular schwannoma. This surgery leads to severe hearing loss or complete deafness on the surgery side as a consequence of damage to the vestibulocochlear nerve. The prevalence of GET after the surgery ranges from 19 to 36\% (Cacace et al., 1994; Biggs and Ramsden, 2002; Baguley et al., 2006).

Whittaker was the first to report GET (Whittaker, 1982, 1983), hypothesizing that GET may be due to cross-modal plasticity, involving axonal sprouting and synapse formation in the CNS. Whittaker argued that regeneration takes place: fibers from the para-abducens nucleus, abducens nucleus, or median longitudinal fasciculus invade the cochlear nucleus $(\mathrm{CN})$, leading to activation of the auditory pathway.

The modulatory character of GET offers a unique opportunity to study the relation between tinnitus and brain activity with functional magnetic resonance imaging (fMRI), which depends on functional contrasts between multiple states of brain activation. Moreover, most GET patients have (nearly) normal hearing at the ear contralateral to the surgery, allowing for psychoacoustic evaluation of the tinnitus characteristics by matching external sounds at the hearing ear to the tinnitus heard in the deaf ear (Cacace et al., 1994; Giraud et al., 1999).

So far, results of three small imaging studies on GET have been published (Cacace et al., 1995; Giraud et al., 1999; Lockwood et al., 2001). These studies showed that tinnitus is accompanied by increased activity in the cerebrum as well as brainstem, which 


$$
\begin{aligned}
& \text { Right ear of left surgery subjects } \\
& \text { Left ear of right surgery subjects }
\end{aligned}
$$
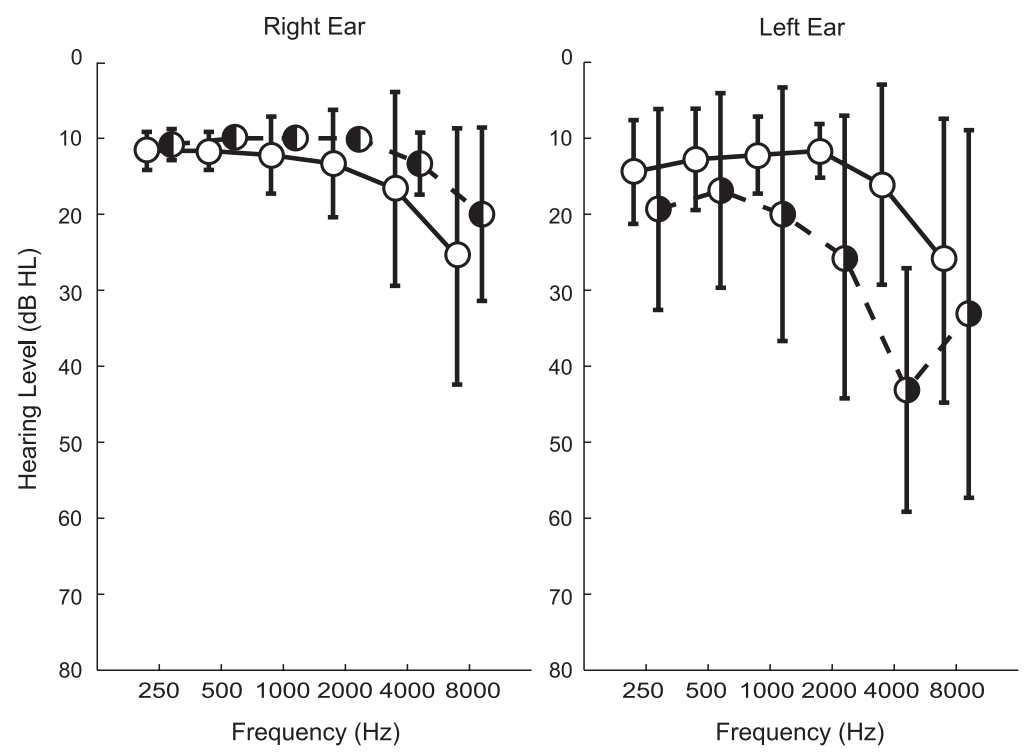

Figure 1. Mean audiograms for the controls $(n=9)$, and the tinnitus subjects who underwent surgery on the left $(n=$ 8 ) or right side $(n=10)$. For tinnitus subjects, only the audiograms of the ear contralateral to the surgery are shown. $0 n$ the side of surgery, the patients were completely deaf, except two, for whom thresholds were between 90 and $120 \mathrm{~dB} H \mathrm{~L}$ (Hearing Level). Error bars indicate SDs.

agrees with hypotheses of hyperactivity in the auditory system (Roberts et al., 2010, Kaltenbach, 2011).

In the present study, psychoacoustic attributes of GET are investigated along with the corresponding neural activity. The spatial resolution of fMRI and the large group of subjects allowed us to identify small changes of activity in the brainstem, thalamus, and cortex, and to relate these changes to loudness modulation of the tinnitus percept. Since the neural activity related to GET is hypothesized to originate in the $\mathrm{CN}$, we hypothesized that increased tinnitus loudness would correspond to increased activity throughout the auditory pathway.

\section{Materials and Methods}

Subjects. Subjects who perceived GET were recruited through an advertisement on the website of the Dutch society for hearing-impaired patients. Eight males and 10 females (aged $51 \pm 8$ years, mean \pm SD) were included. Of all tinnitus subjects, 8 had undergone vestibular schwannoma removal on the left side and 10 on the right side. All GET subjects could modulate or evoke their tinnitus by sustained gaze in a direction deviating from the central axis of the eyes. In addition, nine control subjects (five males and four females; $51 \pm 10$ years) were enrolled. The controls had no tinnitus, hearing complaints, or known neurological diseases. None of the subjects reported any psychiatric history. The study was approved by the local medical ethics committee and written informed consent was obtained from each participant.

Psychoacoustics. Standard pure-tone audiometry was performed in the tinnitus subjects as well as the controls. The hearing thresholds were obtained by using an AC440 type audiometer and TDH-39 headphones. The mean audiogram per group is shown in Figure 1. The control subjects had a mild sensorineural high-frequency hearing loss, despite the absence of any hearing complaints. All tinnitus subjects were interviewed about the characteristics of their GET by means of a questionnaire. Questions were asked about the time interval between surgery and participation in the study, the presence of pre-existing tinnitus before surgery, differences between the tinnitus before and the tinnitus after surgery when looking straight ahead, and the first time the subject noticed the GET (Table 1). Additional information about the diameter of the tumor at the time of surgery, the gradation of the lesion of the facial nerve, and the type of surgery was obtained from clinical files.

All tinnitus subjects, but not the control subjects, performed a tinnitus-matching task for six gaze directions: Central, Left, Up, Right, Down, and Max. Max was defined as the gaze direction that induced the largest (maximal) subjective change in tinnitus, which could be one of these eight directions: Left, Upper Left, Up, Upper Right, Right, Lower Right, Down, or Lower Left (Fig. 2a). The subjects were instructed to gaze in the indicated direction as far as they could maintain for about 10 seconds. The stimuli that were presented to match the tinnitus were presented to the unaffected ear opposite the side of the surgery only.

Initially, the subjects were asked to indicate whether their tinnitus during central gaze most resembled a tone, a narrowband (1/3 octave) noise, or a wideband noise. Next, tinnitus matching was performed for the six gaze directions. If a tone or narrowband noise was chosen to most closely resemble the tinnitus, the procedure was started with a corresponding sound of $1 \mathrm{kHz}$ center frequency, presented at $10 \mathrm{~dB}$ above hearing threshold. This sound was adjusted in frequency, using a step size of $1 / 8$ octave, until subjectively the best matching frequency was reached. Next, the sound level was adjusted in steps of $5 \mathrm{~dB}$ until the loudness matched the tinnitus loudness most closely. Starting from the resulting best matching sound, the frequency and loudness matching procedures were subsequently repeated with step sizes of $1 / 16$ th octave and $1 \mathrm{~dB}$, respectively. If the subject matched the tinnitus with a wideband noise, only the loudness match was performed. To express the intensity level of the matched sound in $\mathrm{dB} S \mathrm{SL}$, the hearing threshold for the matched sound was measured during central gaze. The hearing threshold was then subtracted from the intensity level of the matched sound.

MRI data acquisition. All imaging experiments were performed using a $3 \mathrm{~T}$ MRI system (Philips Intera; Philips Medical Systems), which was equipped with an eight-channel phased-array (SENSE) head coil. The functional scans consisted of $2926 \mathrm{~ms}$ single-shot $\mathrm{T}_{2}{ }^{*}$-sensitive echo-planar imaging sequences with 502.5 -mm-thick slices (TR $10 \mathrm{~s}$, TE $25 \mathrm{~ms}$, in-plane resolution $1.75 \mathrm{~mm}$, field of view $224 \times 224 \times 125 \mathrm{~mm}^{3}$ ). The scans were acquired in ascending order using an oblique transversal orientation so that the lower part of the pons as well as the upper part of the brain were included. The influence of acoustic scanner noise was reduced using a sparse sampling strategy (Hall et al., 1999; Langers et al., 2005). In our experimental design, there was a $7 \mathrm{~s}$ gap of scanner silence between two successive acquisitions. For each subject, three runs of 76 acquisitions were performed. Additionally, a $3 \mathrm{D}$ high-resolution $\mathrm{T}_{1}$-weighted fast-field echo scan was acquired to serve as anatomical reference.

Scanning paradigm. The paradigm included the same six gaze conditions that were used during the psychoacoustic experiment (Central, Left, Up, Right, Down, and Max). For the Max condition, the controls were instructed to look in a direction corresponding to one of the Max directions reported by the tinnitus subjects. An additional condition (Sound) was included that comprised an acoustic stimulus. The purpose of the condition Sound was to be able to delineate the auditory cortex. A bilateral 90 dB SPL dynamic rippled sound (Langers et al., 2005), which was presented while the subjects were looking straight ahead, was never presented while the subjects were performing the gazing task. The spectrum of a dynamic ripple is based on pink noise, but contains spectrotemporal modulations. The stimuli were centered $\sim 1 \mathrm{kHz}$, with a bandwidth of five octaves, a spectral modulation 
Table 1. Tinnitus characteristics and surgery information of all GET subjects

\begin{tabular}{|c|c|c|c|c|c|c|c|c|c|}
\hline Subject & $\begin{array}{l}\text { Surgery } \\
\text { side }\end{array}$ & $\begin{array}{l}\text { Months since surgery } \\
\text { (years; months) }\end{array}$ & $\begin{array}{l}\text { Tinnitus prior } \\
\text { to surgery? }\end{array}$ & $\begin{array}{l}\text { Change of tinnitus } \\
\text { severity since surgery }\end{array}$ & $\begin{array}{l}\text { GET first noticed } \\
\text { (months since surgery) }\end{array}$ & $\begin{array}{l}\text { Hearing loss } \\
\text { ipsilateral (dB HL) }\end{array}$ & $\begin{array}{l}\text { n. VII paresis } \\
\text { (House-Brackmann) }\end{array}$ & $\begin{array}{l}\text { Tumor } \\
\text { diameter }(\mathrm{cm})\end{array}$ & $\begin{array}{l}\text { Surgery } \\
\text { type }\end{array}$ \\
\hline 1 & Left & $20 ; 8$ & No & + & $<1$ & $>120$ & VI & $?$ & $?$ \\
\hline 2 & Left & $22 ; 0$ & Yes & $=$ & ? & $>120$ & III & $?$ & RS \\
\hline 3 & Right & $1 ; 1$ & Yes & $\uparrow \uparrow$ & $?$ & $>120$ & $\|$ & 1.2 & $\mathrm{TL}$ \\
\hline 4 & Left & $19 ; 10$ & Yes & $\uparrow$ & $<1$ & $>120$ & V & 4.0 & $?$ \\
\hline 5 & Right & $11 ; 3$ & No & + & 10 & $>120$ & $\|$ & $?$ & RS \\
\hline 6 & Left & $0 ; 5$ & Yes & $\downarrow$ & 1 & $>120$ & $\|$ & 2.5 & RS \\
\hline 7 & Right & $31 ; 4$ & No & + & $<1$ & $>120$ & I & $?$ & RS \\
\hline 8 & Right & $7 ; 7$ & Yes & $\uparrow$ & $?$ & $>120$ & III & 3.0 & RS \\
\hline 9 & Right & $13 ; 11$ & Yes & $\uparrow \uparrow$ & $?$ & $>120$ & IV & $?$ & $?$ \\
\hline 10 & Left & $3 ; 0$ & Yes & $\uparrow \uparrow$ & 0 & $>90$ & V & $?$ & RS \\
\hline 11 & Left & $5 ; 10$ & No & + & $?$ & $>120$ & I & 3.0 & $\mathrm{TL}$ \\
\hline 12 & Left & $4 ; 4$ & Yes & $\uparrow \uparrow$ & $?$ & $>120$ & $?$ & $?$ & $?$ \\
\hline 13 & Right & $2 ; 3$ & Yes & $\uparrow$ & $>1$ & $>120$ & I & $?$ & $?$ \\
\hline 14 & Right & $6 ; 7$ & No & + & $?$ & $>120$ & I & $?$ & $?$ \\
\hline 15 & Left & $1 ; 1$ & Yes & $\uparrow$ & $?$ & $>120$ & 1 & $?$ & $?$ \\
\hline 16 & Right & $6 ; 9$ & Yes & $\uparrow \uparrow$ & $?$ & $>90$ & VI & 2.5 & $\mathrm{TL}$ \\
\hline 17 & Right & $6 ; 6$ & Yes & $\uparrow \uparrow$ & $?$ & $>120$ & IV & $?$ & RS \\
\hline 18 & Right & $10 ; 2$ & No & & 0 & $>120$ & V & $?$ & $?$ \\
\hline
\end{tabular}

${ }^{+}$, Tinnitus arose after surgery; $\downarrow$, tinnitus has become less severe; $=$, tinnitus remained unchanged; $\uparrow$, the tinnitus worsened; $\uparrow \uparrow$, the tinnitus worsened a lot; I to VI, House-Brackmann grading scale ranging from normal to total; $\mathrm{TL}$, translabyrinthe approach; RS, retrosigmoid approach; ?, not known.

a Gaze directions

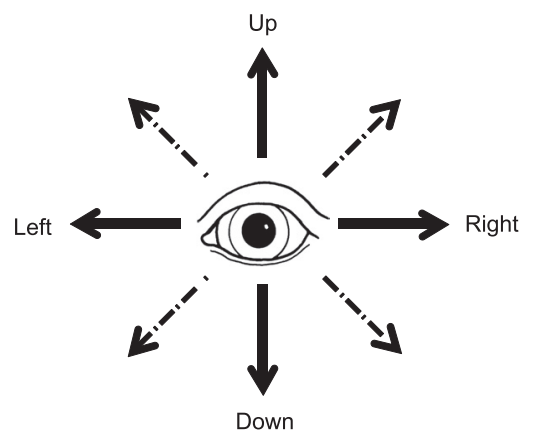

d Bandwidth

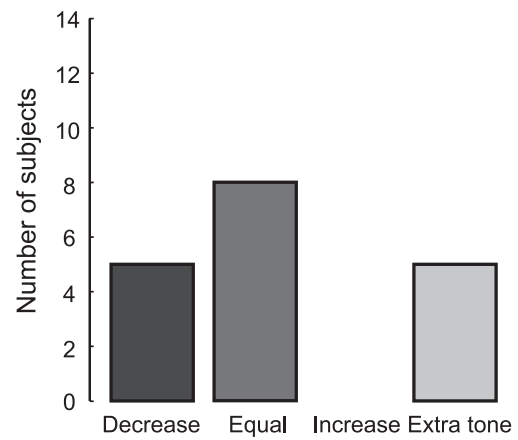

b Gaze direction max tinnitus

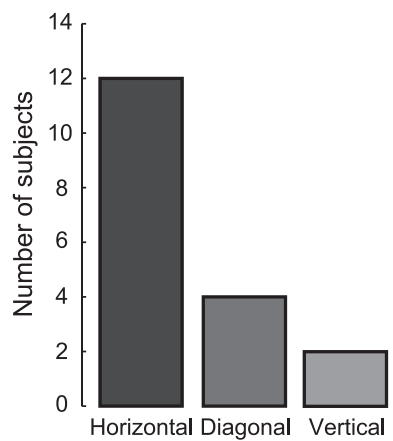

e Loudness

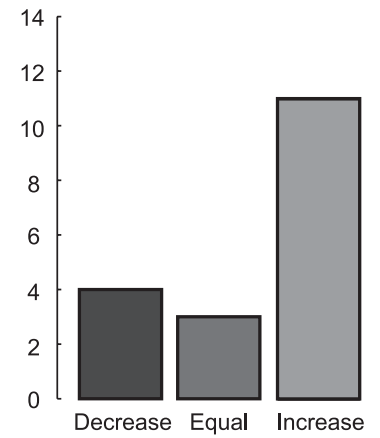

C Gaze lateralization max tinnitus

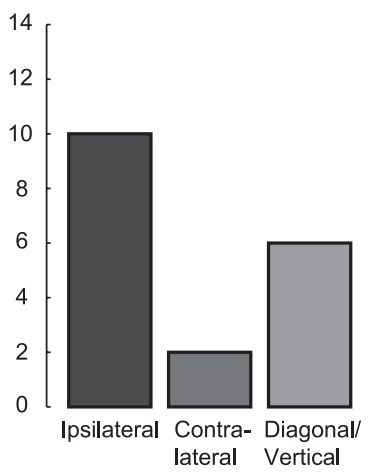

f Pitch

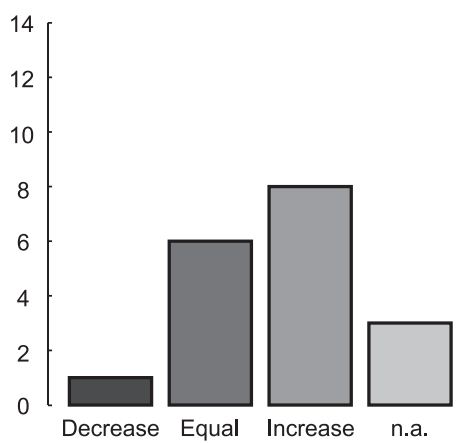

Figure 2. The changes to tinnitus were investigated for five gaze directions (a): Left, Right, Up, Down (solid arrows), and the direction evoking the maximum subjective change of tinnitus (one of the solid or dashed arrows). Histograms showing the characteristics of the perceived tinnitus changes are shown in $\boldsymbol{b}-\boldsymbol{f} \cdot \boldsymbol{b}$, Shows the gaze direction with largest effect on the tinnitus. Gaze lateralization relative to surgery side with largest effect on the tinnitus is shown in c. Changes in, respectively, bandwidth, loudness, and pitch for the maximum tinnitus percept are shown in $\boldsymbol{d}-\boldsymbol{f}$. n.a., Not applicable.

density of 0.1 cycle per octave, a modulation amplitude of $15 \%$, and a temporal modulation frequency of five cycles per second, which produces a $5 \mathrm{~Hz}$ intensity modulation. Sound was presented by means of MR-compatible electrodynamic headphones (MR Confon GmbHBaumgart et al., 1998) connected to a standard PC with soundcard. Under the headset, subjects wore foam ear plugs to further attenuate the acoustic noise produced by the scanner.

An instruction was delivered to the subject by means of a symbol shown centrally on a projection screen. The symbol consisted of a fixa- tion cross for the Sound and Central conditions, the text "MAX" for the Max condition, or an arrow in the appropriate direction for the Left, Up, Right, and Down gaze conditions. The subject performed the task indicated by the symbol during scanning and for the duration of the subsequent silent period. The subject was instructed to look back at the screen when scanner noise was heard, signaling the imminent start of the next trial. In each run, the Sound, Central, Left, Up, Right, Down and Max conditions occurred 13, $13,10,10,10,10$, and 10 times, respectively, resulting in a total of 76 trials that were presented in a pseudorandom order. 
To monitor whether the task was performed correctly, a $50 \mathrm{~Hz} \mathrm{MR}$ compatible eye tracker was used, connected via fiber optics to a PC running iViewX software (version 1.0; SMI). A mirror relayed the image of the eye to the infrared camera of the eye tracker, mounted at the foot of the scanner bed. If the task was not well performed, the corresponding scan was excluded from analysis. Task performance was considered inadequate when the subject did not gaze in the specified direction or excessively moved the eyes during the task. This only happened in 6 of 27 subjects, resulting in the exclusion of 15 scans.

Data processing and statistical analysis. The MR images were analyzed using SPM8 (Functional Imaging Laboratory, The Wellcome Department of Imaging Neuroscience, London, http://www.fil.ion.ucl.ac. $\mathrm{uk} / \mathrm{spm} /$ ) running under MATLAB R2010a (MathWorks). The functional images were first corrected for motion using rigid body realignment. During this correction procedure, the orbital regions were masked to minimize the influence of artifacts due to eye movements. Thereafter, the images were spatially coregistered with the $T_{1}$-weighted high-resolution anatomical image, and all images were normalized into Montreal Neurological Institute stereotaxic space. To improve the signal-to-noise ratio, the functional data were spatially smoothed using an isotropic Gaussian kernel with a full-width at half-maximum of $8 \mathrm{~mm}$. A logarithmic transformation was performed to express the signal measures in percentage signal change (Langers et al., 2012).

A multiple regression analysis was performed for each subject. The regression model included all seven task conditions and the realignment parameters, as well as a linear term to model drift within each run. Subsequently, the contrast images of the six conditions of interest (Sound, Left, Up, Right, Down and Max), relative to the silent straight ahead condition (Central), were fed into a mixed effects group analysis comprising a factorial design with factors for stimulus condition, subject, and group membership (i.e., surgery right, surgery left, and controls).

Because of the comorbid unilateral deafness, one-sample $t$ tests were used to determine the significant responses to the Sound condition for the three subject groups separately. With regard to the five gaze directions, independent samples $t$ tests were performed to calculate the significances of differences between pairs of groups (right surgery, left surgery, and controls). Because no substantial differences were found, the responses to the five gaze conditions were subsequently assessed by performing a one-sample $t$ test on the responses of all subjects. A familywise error corrected confidence threshold of $p<0.05$ was applied for statistical testing.

Region-of-interest analysis. In addition to the voxel-by-voxel multiple regression analysis, a region-of-interest (ROI) analysis was performed. Gaze-evoked responses were assessed in eight anatomical areas comprising parts of the auditory and visual pathways. The $\mathrm{CN}$, inferior colliculi (IC), and superior colliculi (SC) were defined per subject based on their anatomical image. The ROI consisting of the $\mathrm{CN}$ included the lower posterior part of the brainstem and was drawn near the cerebellopontine angle (Hawley et al., 2005). The colliculi were drawn on the easily identifiable superior and inferior protrusions of the quadrigeminal plate on the posterior side of the midbrain. The medial geniculate bodies (MGB) and lateral geniculate bodies (LGB) were selected according to the WFU pickatlas (Maldjian et al., 2003). The bilateral auditory cortex (AC) was defined by means of the regression outcomes; this ROI comprised all voxels in the temporal lobe that were activated by the condition Sound in the control group (see Results). Furthermore, a gaze activated area (GAZE+) and a gaze deactivated area (GAZE-) ROI were included. These two areas comprised all voxels that were activated or deactivated by gaze, respectively (see Results).

To assess the response lateralization to gaze in all defined ROIs, we also calculated the average activation separately for the left and right ROIs, respectively. For each group (i.e., left surgery, right surgery, and controls), the average activation in each region was calculated for all gaze directions against central fixation.

In each ROI and for both the complete GET group (i.e., left and right surgery combined) and the control group, the average gaze-evoked activation was calculated across the contrast images Left, Up, Right, Down, and Max. In these ROI analyses, the significance of the response relative to baseline (i.e., central fixation) and the difference between the GET group and control subjects were assessed by two-tailed $t$ tests.

Next, the contrast images of the various gaze directions in the tinnitus subjects were subdivided into three sets according to the loudness increase of the tinnitus perceived by the subject and measured during the tinnitusmatching task. This subdivision was performed to determine whether the perceived loudness is represented in the activation of the respective bilateral ROIs. The first set, labeled " $\leq 0 \mathrm{~dB}$," contained all the contrasts for which the subjects did not perceive any loudness increase. This set also contained all contrasts for which decreases in loudness were perceived. A second set labeled " $1-5 \mathrm{~dB}$ " contained all the contrasts for which the subjects gazed in directions that induced loudness increases up to and including $5 \mathrm{~dB}$. The third set, labeled " $>5 \mathrm{~dB}$," contained all the direction contrasts that caused loudness increases exceeding $5 \mathrm{~dB}$.

Bootstrapping tests (Wu, 1986; Liu, 1988) were performed to test whether the responses in each of the sets significantly differed from zero, respectively. For each set, the random inversion of response amplitudes involved in this procedure was iterated 50,000 times The 50,000 estimates were used to derive null distributions to assess whether significance was reached $(p<0.05)$.

In addition, nonparametric permutation tests (Good, 2002; Nichols and Holmes, 2002) were performed to test whether significant differences in responses between each set of the GET patients and the controls existed. To test the significance of these set differences, the subjects were first randomly reassigned to the two subject groups (controls and GET patients), while retaining the original group sizes. Second, for the subjects assigned to the GET group, the responses were additionally permuted across the perceptual sets $(\leq 0 \mathrm{~dB}, 1-5 \mathrm{~dB}$, and $>5 \mathrm{~dB})$. The resampling of the 135 responses was iterated 50,000 times. For each iteration, the difference between the means was calculated. The 50,000 estimates were combined to obtain a null distribution that was used to assess whether significance was reached $(p<0.05)$.

\section{Results}

\section{Psychoacoustics}

The characteristics of the subjects with GET are listed in Table 1. The majority of the tinnitus subjects were deaf postoperatively on the side of the surgery; however, in two subjects (\#10 and \#16) hearing remained partially preserved. Removal of the tumor was often accompanied by a paresis of the cranial nerve VII. Of all subjects, 12 already perceived tinnitus before the tumor surgery. The tinnitus severity had increased in all subjects since the surgery, except in one subject who experienced a decrease and one subject for whom the severity did not change. Ten of the 18 tinnitus subjects did not remember at which point in time they first noted their tinnitus could be modulated by gaze. The onset time of the remaining tinnitus subjects ranged from immediately after surgery to 10 months postoperatively.

All tinnitus subjects reported to hear their tinnitus in the ear on the side of the surgery, regardless of gaze direction. Tinnitus modulation due to gaze was similar in a darkened room as compared with a normally lighted room. The effect of gaze was similar when the head was fixed while the eyes changed position, as when the subjects fixated on a cross while turning their head. In other words, the effect on the tinnitus was mainly determined by the orientation of the eyes relative to the skull. In 15 subjects, the effect on tinnitus sustained as long as the gaze remained fixed. Three subjects described that the tinnitus changed only during eye movement, whereas the tinnitus went back to its baseline as soon as the eye position was maintained.

The effect of gaze on the tinnitus characteristics depended on the gaze direction. A systematic evaluation of the effect of gaze while the head was fixed showed a diverse pattern of tinnitus modulations across subjects (Table 2; Fig. 2). Based on the nature of these modulations, tinnitus subjects were classified in five categories (Table 2). In three subjects (Category I), gaze resulted in 
Table 2. Tinnitus characteristics for various gaze directions

\begin{tabular}{|c|c|c|c|c|c|c|c|c|c|c|c|c|c|c|c|c|c|c|c|}
\hline \multirow[b]{2}{*}{ Subject } & \multicolumn{3}{|c|}{ Center } & \multicolumn{3}{|c|}{ Left } & \multicolumn{3}{|l|}{ Up } & \multicolumn{3}{|c|}{ Right } & \multicolumn{3}{|c|}{ Down } & \multicolumn{4}{|l|}{$\operatorname{Max}$} \\
\hline & B & $\mathrm{F}$ & L & B & $\mathrm{F}$ & L & B & $\mathrm{F}$ & L & B & $f$ & L & B & $\mathrm{F}$ & $\mathrm{L}$ & D & B & $\mathrm{F}$ & L \\
\hline \multicolumn{20}{|c|}{ I. Extra sound } \\
\hline 1 & - & - & - & T & 1.6 & 20 & T & 6.5 & 20 & $T$ & 1.6 & 20 & $T$ & 7 & 20 & LD & $\mathrm{T}$ & 6.5 & 22 \\
\hline 2 & WB & n.a. & 13 & T & 5 & 5 & $=$ & $=$ & $=$ & T & 5 & 5 & $=$ & $=$ & $=$ & $\mathrm{L}$ & T & 5 & \\
\hline 3 & WB & n.a. & 25 & $\mathrm{~T}$ & 9 & 5 & $=$ & $=$ & $=$ & $=$ & $=$ & $=$ & $=$ & $=$ & $=$ & L & $\mathrm{T}$ & 9 & \\
\hline \multicolumn{20}{|c|}{ II. Loudness change } \\
\hline 4 & WB & n.a. & 18 & $=$ & $=$ & 20 & $=$ & $=$ & $=$ & $=$ & $=$ & 19 & $=$ & $=$ & $=$ & L & $=$ & $=$ & 20 \\
\hline 5 & NB & 4.2 & 10 & $=$ & $=$ & 30 & $=$ & $=$ & 20 & $=$ & $=$ & 35 & $=$ & $=$ & 15 & R & $=$ & $=$ & 35 \\
\hline \multicolumn{20}{|c|}{ III. Loudness and pitch change } \\
\hline 6 & T & 8 & 10 & $=$ & 9 & 0 & $=$ & 10 & 0 & $=$ & 9 & 0 & $=$ & $=$ & $=$ & L & $=$ & 9 & \\
\hline 7 & NB & 2 & 1 & $=$ & 7.2 & 4 & $=$ & 7.2 & 3 & $=$ & 7.2 & 5 & $=$ & 7.2 & 2 & $\mathrm{R}$ & $=$ & 7.2 & \\
\hline 8 & NB & 1.5 & 15 & $=$ & $=$ & $=$ & $=$ & 2 & 12 & $=$ & $=$ & $=$ & $=$ & $=$ & $=$ & U & $=$ & 2 & 12 \\
\hline 9 & $T$ & 1.4 & 19 & $=$ & 1.5 & 22 & $=$ & 1.6 & 26 & $=$ & 1.3 & 22 & $=$ & 1 & 22 & U & $=$ & 1.6 & 26 \\
\hline 10 & T & 1.7 & 36 & $=$ & 2.0 & 49 & $=$ & $=$ & $=$ & $=$ & $=$ & $=$ & $=$ & $=$ & $=$ & L & $=$ & 2.0 & 49 \\
\hline 11 & T & 4.9 & 17 & $=$ & 6.3 & 25 & $=$ & $=$ & $=$ & $=$ & 6.3 & 18 & $=$ & 6 & 18 & L & $=$ & 6.3 & 25 \\
\hline \multicolumn{20}{|c|}{ IV. Loudness and bandwidth change } \\
\hline 12 & WB & n.a. & 20 & NB & 9.5 & 5 & NB & 2.5 & $=$ & NB & 16 & 16 & $=$ & $=$ & $=$ & $\mathrm{R}$ & NB & 16 & 16 \\
\hline 13 & WB & n.a. & 0 & $=$ & $=$ & $=$ & NB & 2.4 & 4 & NB & 2.4 & 10 & $=$ & $=$ & $=$ & $\mathrm{R}$ & NB & 2.4 & 10 \\
\hline 14 & WB & n.a. & 5 & $=$ & $=$ & $=$ & $=$ & $=$ & $=$ & NB & 10 & 15 & $=$ & $=$ & $=$ & $\mathrm{R}$ & NB & 10 & 15 \\
\hline 15 & NB & 4.4 & 21 & $=$ & $=$ & $=$ & $=$ & $=$ & $=$ & $=$ & $=$ & $=$ & $=$ & $=$ & $=$ & $\mathrm{RD}$ & $T$ & 1.5 & 15 \\
\hline 16 & NB & 0.125 & 10 & NB & 0.16 & 15 & $=$ & $=$ & $=$ & $\mathrm{T}$ & 6 & 25 & $=$ & $=$ & $=$ & $\mathrm{R}$ & T & 6 & 25 \\
\hline \multicolumn{20}{|c|}{ V. Complex } \\
\hline \multirow[t]{2}{*}{17} & NB & 9.5 & 2 & $=$ & $=$ & 5 & $=$ & $=$ & $=$ & $=$ & $=$ & $=$ & $=$ & $=$ & 4 & RU & $=$ & $=$ & $=$ \\
\hline & - & - & - & $\mathrm{T}$ & 9.5 & 0 & $=$ & $=$ & $=$ & $\mathrm{T}$ & 9.5 & 3 & $=$ & $=$ & $=$ & RU & $\mathrm{T}$ & 9.5 & 5 \\
\hline \multirow[t]{2}{*}{18} & NB & 0.2 & 5 & $=$ & $=$ & 10 & $=$ & $=$ & 10 & $=$ & $=$ & $=$ & $=$ & $=$ & $=$ & LU & $=$ & $=$ & 10 \\
\hline & - & - & - & T & 2 & 0 & $=$ & $=$ & $=$ & $=$ & $=$ & $=$ & $=$ & $=$ & $=$ & LU & $\mathrm{T}$ & 2.5 & 15 \\
\hline
\end{tabular}

The column headers indicate the gaze directions. Per gaze direction, the bandwidth (B), matched pitch ( $\mathrm{F}$, $[\mathrm{kHz}])$, and matched loudness level (L, [dB SL]) are denoted. Bandwidth was tonal (T), narrowband (NB), or wideband (WB). The column Max indicates the gaze direction (D) and tinnitus characteristics evoking the maximum tinnitus percept in the subjects. Subjects are grouped with respect to the most prominent effect of gaze on tinnitus. I. Extra sound, an (extra) sound arose by eye movements. In subjects \#2 and \#3, the sound heard when looking straight ahead persisted for all gaze directions. II. Loudness change, only the loudness of the tinnitus changed by eye movements. III. Loudness and pitch change, both the loudness and the pitch of the tinnitus changed by eye movements. IV. Loudness and bandwidth change, both the loudness and the bandwidth of the tinnitus changed by eye movements. In subjects \#12 and \#16, the pitch of the tinnitus changed as well. V. Complex, the changes in the tinnitus induced by gaze corresponded to an alteration in tinnitus loudness. In addition to this alteration, a new tone appeared during performing the eye movements. The direction (D) of the gaze direction that gave a maximum increase of tinnitus loudness is indicated as $L$, left; $L D$, left down; $L U$, left up; $R$, right; $R D$, right down; $R U$, right up; $U$, up. =, no effect of gaze on the particular tinnitus characteristic; n.a., not applicable.

an extra sound, either in addition to a baseline tinnitus that was present when looking straight ahead (\#2 and \#3), or in the absence of such a baseline tinnitus (\#1). The additional sound was perceived on the same side as the original tinnitus. All other subjects described an effect of gaze on the loudness, the pitch, and/or the bandwidth of their existing tinnitus. Two subjects experienced a loudness change, while the apparent bandwidth and the pitch remained unchanged (Category II). A tinnitus loudness change was accompanied by a pitch change in six subjects (Category III), and by an additional bandwidth change in five subjects (Category IV). Two subjects described perceiving two distinct tinnitus sounds, which were differently affected by gaze (Category V).

Fig. 2, $b-f$, shows various characteristics regarding the maximally modulated tinnitus percept. Figure $2, b$ and $c$, is related to gaze characteristics, whereas Figure $2, d-f$, is related to tinnitus characteristics. Despite the diversity of effects, some general trends could be observed: the largest effect on tinnitus loudness was most frequently described for a horizontal gaze direction (Fig. 2b), and most often this maximum effect occurred when the subject gazed to the side of the surgery (Fig. $2 c$ ). Four subjects experienced the largest effect on tinnitus loudness when gazing diagonally: two when gazing up/down ipsilaterally to the side of surgery (\#1 and \#17 in Tables 1 and 2), and two when gazing up/down contralaterally to the side of surgery (\#15 and \#18).

If subjects experienced a change in bandwidth of the tinnitus, it was always a reduction (wideband to narrowband, or narrowband to tonal). Also, the emergence of a new tinnitus sound was always tonal (Fig. 2d). Tinnitus loudness increases occurred more often than decreases (Fig. 2e). Tinnitus pitch increases occurred more frequently than decreases (Fig.2f). Pitch changes were always accompanied by a loudness change. Bandwidth changes were always accompanied by both pitch and loudness changes.

\section{Full brain analysis}

The responses to the bilateral sound stimulus in both the tinnitus group and the controls are displayed in Figure 3. In patients (Fig. $3 a, b)$, auditory cortical activation was largest on the side ipsilateral to the surgery. In addition, activation was visible in the IC ipsilateral to the surgery. In the control group, the left and the right $\mathrm{AC}$ showed similar responses to sound, and activation in bilateral IC and right MGB was observed (Fig. 3c). When relaxing the threshold to $p<0.001$ (uncorrected for multiple comparisons), the left MGB was visible as well.

The brain activation for the different gaze directions, Left, Up, Right, Down, and Max, did not show substantial differences in a comparison between all pairs of subject groups. Therefore, Figure 4 pools the responses of all subjects groups. Horizontal gaze to the left (Fig. $4 a$ ) yielded activation in the contralateral precuneus and extensive deactivation in the primary visual cortex. Vertical gaze upward (Fig. $4 b$ ) resulted in activation dorsally in the occipital lobe and deactivation in the primary visual cortex. Horizontal gaze to the right (Fig. 4c) led to activation in the contralateral precuneus and widespread deactivation in the primary visual cortex. Vertical gaze downward (Fig. $4 d$ ) and gazing to the Max direction (Fig. 4e) resulted in some activation mainly coinciding with the inferior areas of the brain regions that were also activated when looking to the left and the right. The dominant effect of downward gaze and 


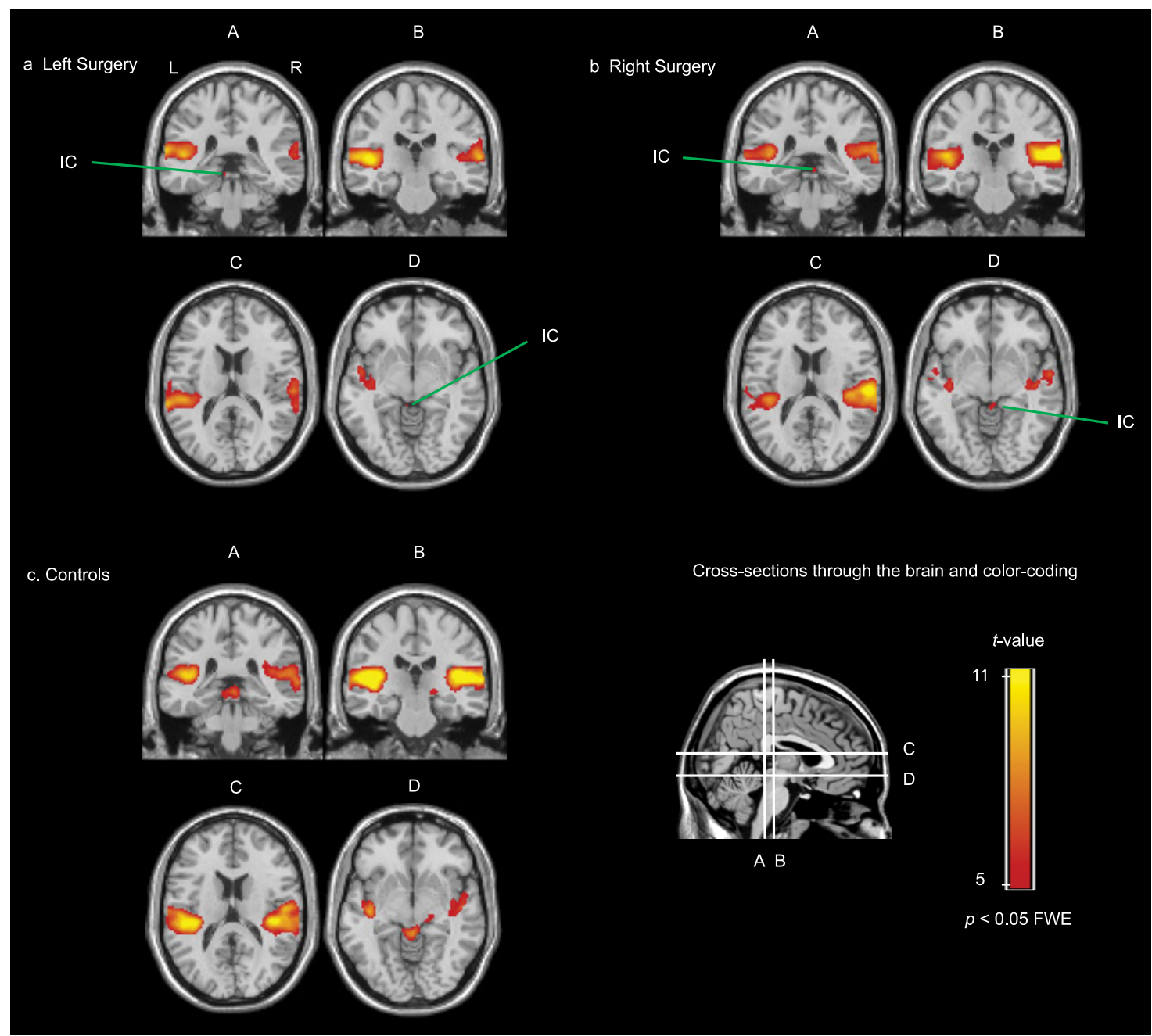

Figure 3. Coronal and transversal cross sections of the brain through the $A C(\boldsymbol{A}-\boldsymbol{D}), \operatorname{MGB}(\boldsymbol{B}), \mathrm{IC}(\boldsymbol{A}, \boldsymbol{D})$, and $\mathrm{CN}(\boldsymbol{A})$ showing significant responses to bilateral $90 \mathrm{~dB}$ SPL dynamic rippled sound in the subjects who underwent surgery on the left side $(n=8 ; \boldsymbol{a})$, the subjects who underwent surgery on the right side $(n=10 ; \boldsymbol{b})$, and the control subjects $(n=9 ; \boldsymbol{c})$. The red-yellow color-coded areas indicate areas with a significant response.

gaze to the Max direction was extensive deactivation in the primary visual cortex. Fig. $4 f$ shows the $t$ values (i.e., significance) of the averaged activation or deactivation across all subjects and gaze directions.

\section{ROI analysis}

In addition to the multiple regression analysis, ROI analyses were performed. The ROIs GAZE + and GAZE - were defined as the occipital areas that responded to peripheral gaze by activation and deactivation, respectively, when averaged across all subjects (Fig. 4f).

Figure 5 shows the responses of the cortical and subcortical auditory and visual areas, averaged across all gaze directions. No distinction between hemispheres was made. Peripheral gaze resulted in deactivation of the auditory cortex in control subjects. The CN, IC, and SC, and the MGB and LGB did not show a significant response in the controls. In tinnitus subjects, the AC showed inhibition but it was significantly reduced compared with that in the controls. In contrast to the response in the controls, the IC showed activation, and the MGB and LGB showed deactivation in the tinnitus subjects. Similar to the controls, there was no response in the $\mathrm{SC}$ and $\mathrm{CN}$ of the tinnitus subjects. The
GAZE + and GAZE - occipital areas were already known to show activation and deactivation, respectively (Fig. $4 f$ ), but were not significantly different between patients and controls.

Figure 6 shows the lateralization of the activations and deactivations in the ROI. Here, the three subject groups were considered separately: controls, subjects with left surgery, and subjects with right surgery.

One possible result that might be expected in this representation includes different patterns of activation for the left and right surgery subjects. Since the tinnitus is perceived on the side of the surgery, one could predict left-right mirrored activation in both patient groups. Figure 6 shows no evidence of such mirrored activation for both tinnitus groups, respectively.

An alternative possibility would be that a specific hemisphere is involved in the tinnitus, regardless of the side of the surgery. The responses in Figure 6 show some evidence of a specific hemisphere lateralization. In both tinnitus groups, the reduced inhibition in the $\mathrm{AC}$ was significant in the left hemisphere, but not in the right hemisphere. In addition, the inhibition of the MGB and LGB was significant in the right hemisphere, but not in the left hemisphere.

As a next step, the ROI responses corresponding with the various gaze directions in the tinnitus subjects were subdivided 


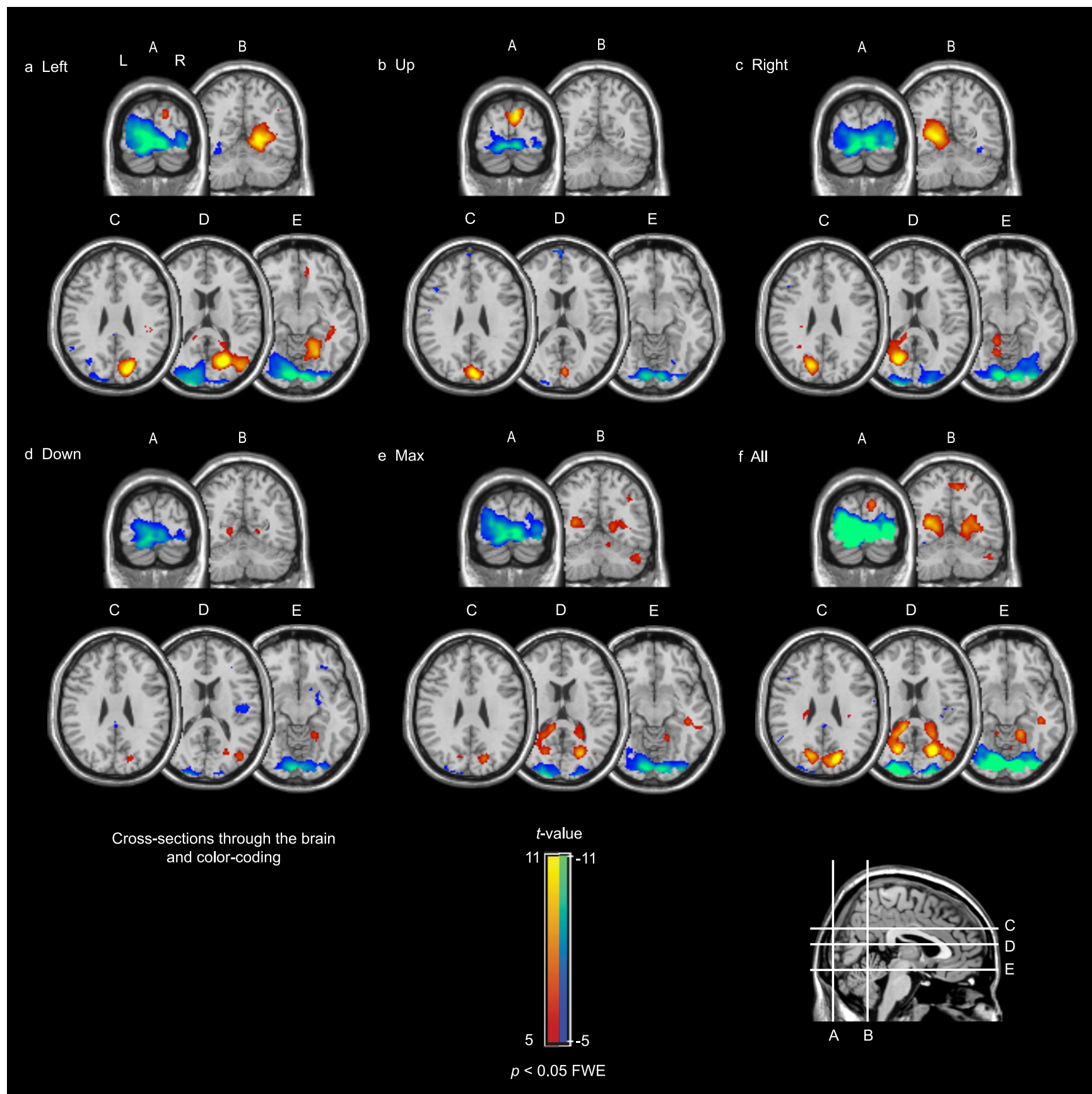

Figure 4. Brain responses to the five peripheral gaze directions, contrasted with gazing straight ahead. The responses of tinnitus and control subjects were combined $(n=27)$. Deactivation was found in primary visual cortex $(\boldsymbol{A}-\boldsymbol{E})$; activation was found in precuneus $(\boldsymbol{a}, \boldsymbol{c}-\boldsymbol{e}$; cross sections $\mathrm{B}-\mathrm{E})$, and dorsally in the occipital lobe $(\boldsymbol{b}$; cross sections $\mathrm{A}, \boldsymbol{C})$. $\boldsymbol{f}$, Shows the average activation and deactivation across all subjects and all gaze directions. The red-yellow color-code indicates areas with a significantly increased activity to gazing; the blue-green color-coded areas indicate areas with a significantly decreased activity.

into three sets according to the loudness increase of the tinnitus perceived by the subject as measured during the tinnitus matching task, and these results were compared with those from the controls. Figure 7 shows the response levels in the bilateral ROIs per set $(\leq 0 \mathrm{~dB}, 1-5 \mathrm{~dB}$, and $>5 \mathrm{~dB}$ ). Two subjects (\#15 and \#16) could not hear their tinnitus modulations during scan time. All their responses were included in the $\leq 0 \mathrm{~dB}$ set. In total, 49, 19, and 22 responses were included in the sets $\leq 0 \mathrm{~dB}, 1-5 \mathrm{~dB}$, and $>5$ $\mathrm{dB}$, respectively (for a total of 90 responses, corresponding to 18 tinnitus subjects gazing in five directions). Of the 49 responses in set $\leq 0 \mathrm{~dB}, 35$ correspond to no perceived loudness change and 14 to a loudness decrease. The number of right/left surgery tinnitus subjects for each mentioned set equaled $7 / 7,5 / 2$, and $5 / 3$, respectively. The number of right surgery, respectively, left surgery subjects exceeds the total number of subjects belonging to both groups, because subjects may have more than one response falling within the various response sets.

The subplots in Figure 7 show various patterns. The bootstrap test showed a significant decrease of the blood-oxygen-leveldependent (BOLD) signal in the AC in the controls and the tinnitus patients. The response levels for the $\leq 0 \mathrm{~dB}$ and $1-5 \mathrm{~dB}$ sets were significantly decreased, but the significance declines with 
Auditory areas

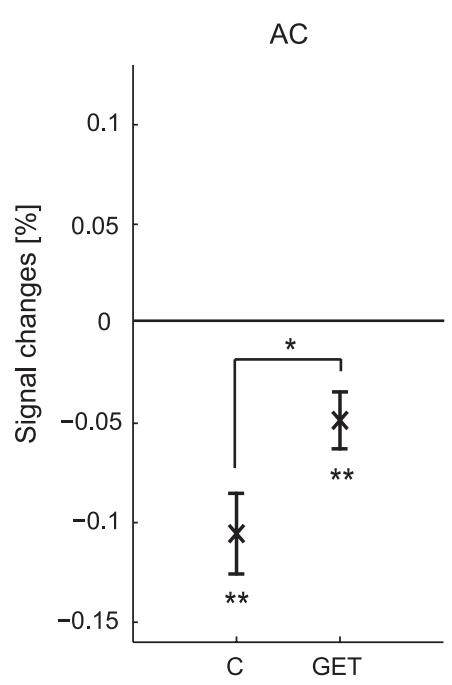

IC

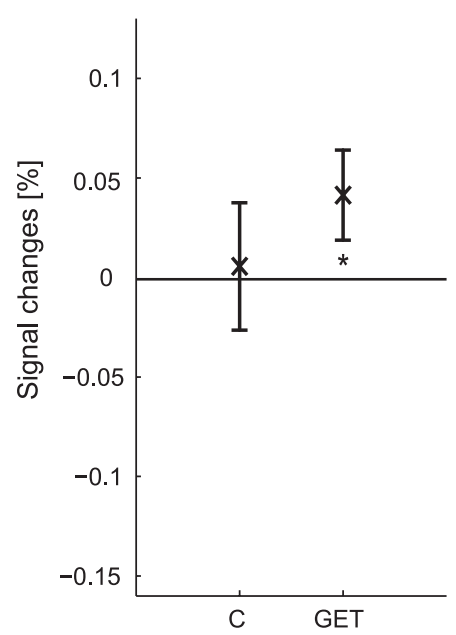

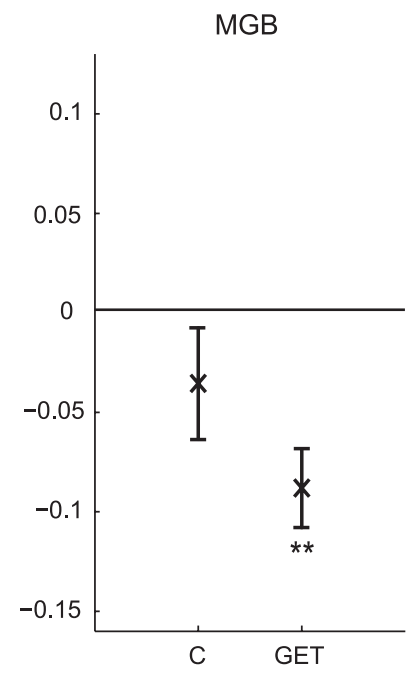

$\mathrm{CN}$

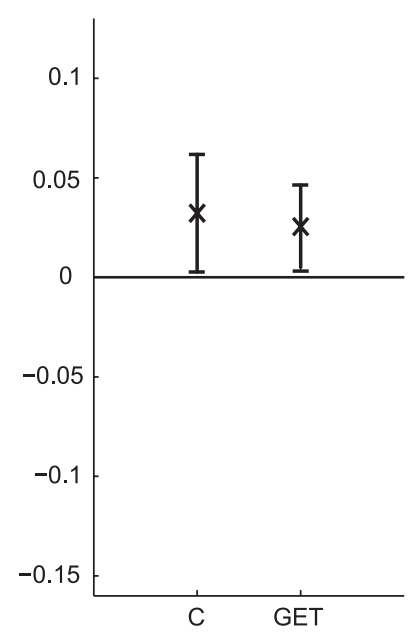

$\begin{array}{ll}* \quad p & <0.05 \\ * * & p<0.001\end{array}$

Visual areas
LGB

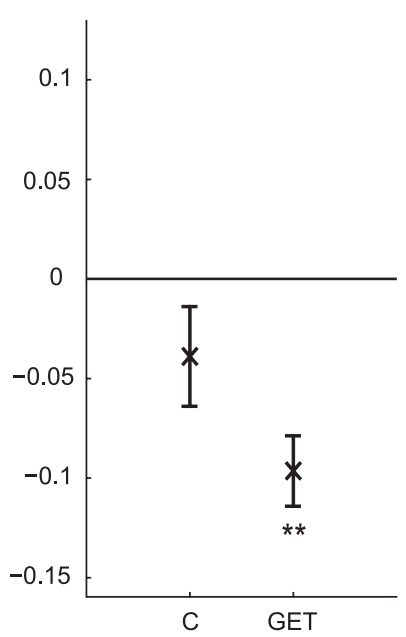

SC

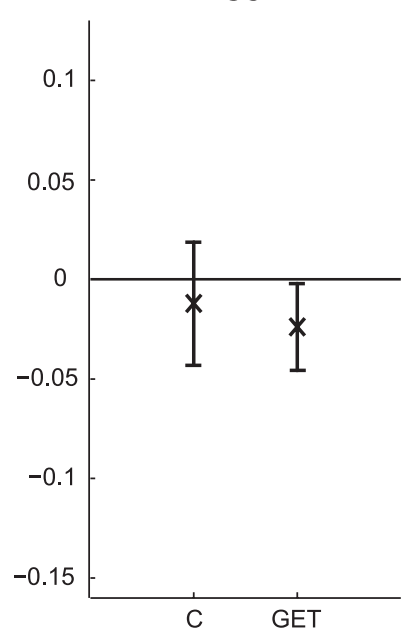

GAZE+

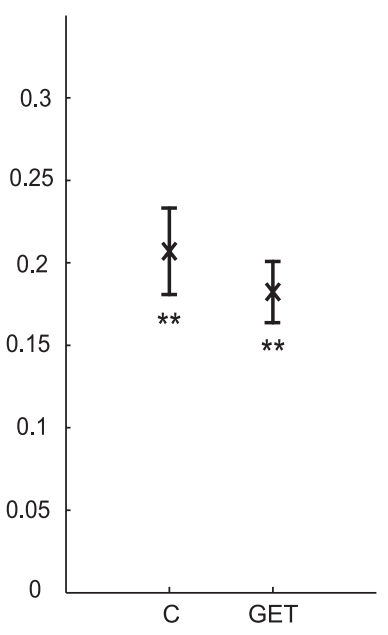

GAZE-

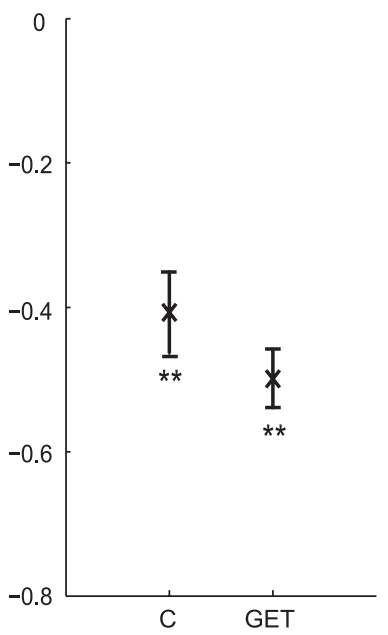

Figure 5. ROI responses to peripheral gaze for the controls $(C ; n=9)$ and the tinnitus subjects (GET; $n=18)$. Left surgery and right surgery subjects were combined. Also, the left and the right hemisphere, as well as the gaze directions, were combined. ROl analyses were performed on bilateral AC, MGB, IC, CN, LGB, and SC, as well as the GAZE + and GAZE - . Error bars indicate the group SEs around the mean. Statistical significances against baseline and differences between the GET group and the control group are represented by asterisks.

increasing tinnitus loudness. For the loudest tinnitus $(>5 \mathrm{~dB})$ the signal decrease was not observed. The three GET sets in the MGB showed deactivation, but this was not related to the tinnitus loudness. The deactivations of the $\leq 0 \mathrm{~dB}$ and $1-5 \mathrm{~dB}$ sets reached significance. Furthermore, significant increases of the IC and CN were detected. The response levels in the sets $1-5 \mathrm{~dB}$ and $>5 \mathrm{~dB}$ of these ROIs showed significant increases compared with baseline. In the LGB, the response levels in the three GET sets showed significant decreases.

Permutation testing revealed a significant difference in means between set $>5 \mathrm{~dB}$ and the controls in the AC. The inhibition in the controls was significant relative to the tinnitus subjects. For the ROI GAZE-, we observed significant decreases for the sets $1-5 \mathrm{~dB}$ and $>5 \mathrm{~dB}$ in the tinnitus group relative to the controls.

\section{Discussion}

The perceptual characteristics of GET and their relation to brain activity were explored. For the gaze maneuvers that changed tinnitus, most often the tinnitus loudness increased, the matched bandwidth decreased or remained unchanged, and the tinnitus pitch usually increased or remained unchanged. An increase in loudness corresponded to an increased BOLD signal in the AC and IC. In tinnitus subjects, peripheral gaze resulted in a deactivation in the MGB; however, the magnitude of the decrease was not related to the tinnitus loudness. To our knowledge this is the first study that shows a relation between tinnitus loudness and simultaneous activity in the midbrain, thalamus, and cortex.

\section{Bandwidth of the tinnitus}

The diversity of perceptual characteristics in our tinnitus subjects (Table 2) is remarkable. Although these subjects had a similar medical history, with removal of a vestibular schwannoma as an important common episode, both the nature of the unmodulated tinnitus and the effect of gaze varied substantially.

Curiously, the stimulus bandwidth that matched the unmodulated tinnitus (column "center" in Table 2) varied across our subjects from wideband noise to tonal. In cases of partial high-frequency hearing loss, the tinnitus pitch has been shown to 


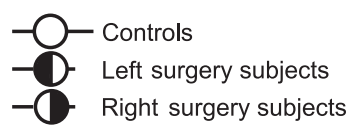

Auditory areas

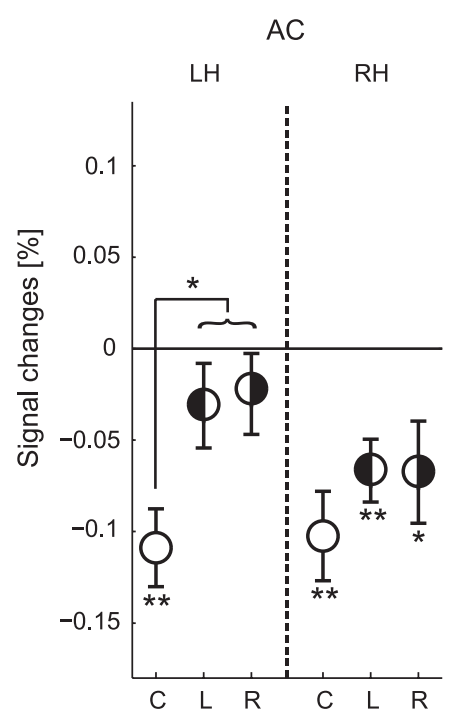

MGB
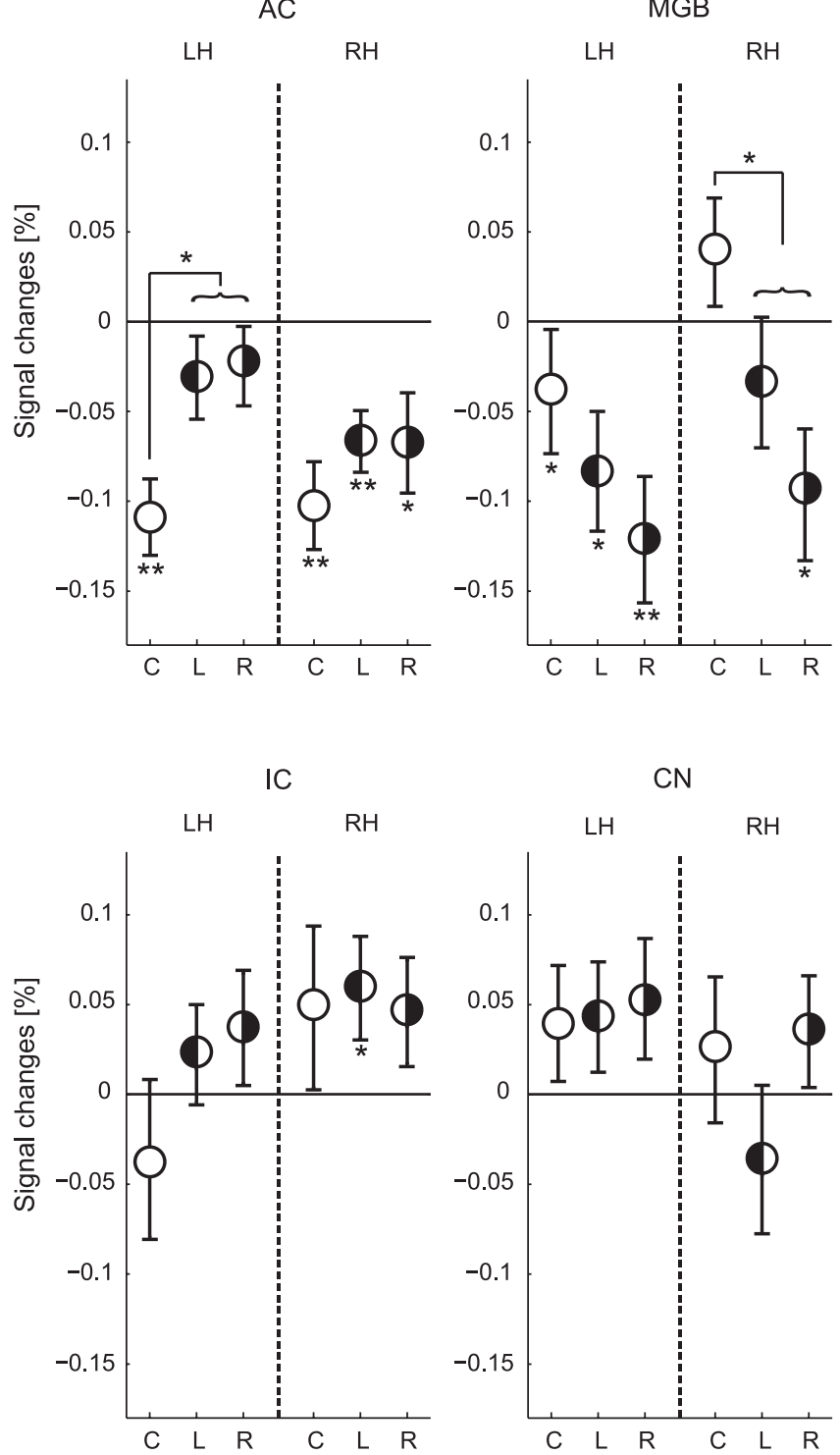

$\mathrm{CN}$

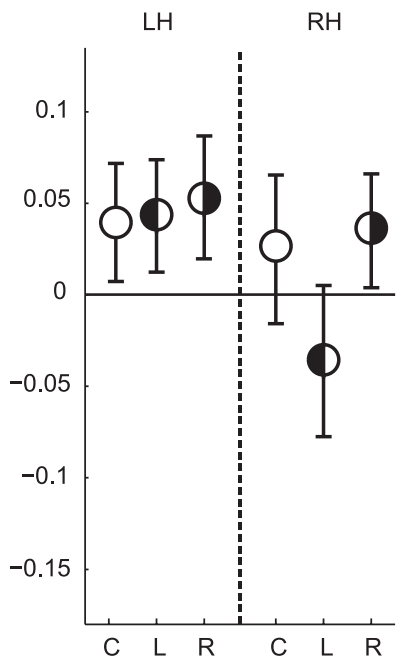

SC

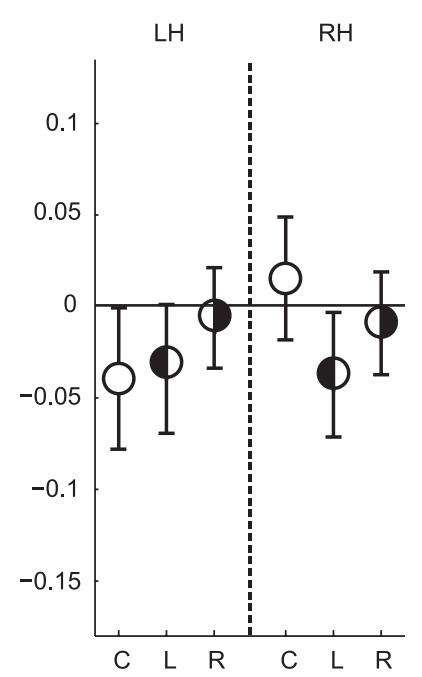

Visual areas
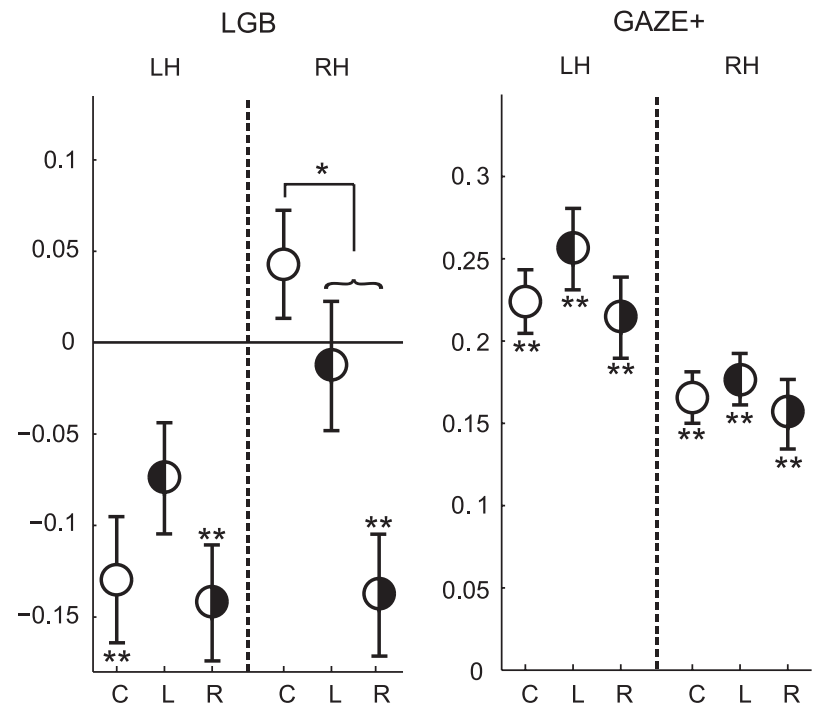

Figure 6. ROI responses to peripheral gaze for the controls $(C ; n=9)$ and the left surgery $(L ; n=8)$ and right surgery $(R ; n=10)$ tinnitus groups. In contrast to Figure 5 , the left and right surgery subjects and the left and the right hemispheres are shown separately. ROl analyses were performed on unilateral AC, MGB, IC, CN, LGB, and SC, as well as the GAZE + and GAZE - . Error bars indicate the group SEs around the mean. Statistical significances against baseline and differences between the GET group and the control group are represented by asterisks. LH, left hemisphere; RH, right hemisphere.

correspond to the frequency range of impaired hearing (Norena et al., 2002; Sereda et al., 2011), or the edge frequency of the hearing loss (König et al., 2006; Moore et al., 2010). These tinnitus percepts have been interpreted to be the consequence of partial deafferentation: due to the tonotopic organization of the auditory system, loss at certain frequencies is expected to result in tinnitus that is related to the hearing loss frequencies. Our subjects had (nearly) complete loss of auditory function in the surgery ear. Thus, it would be expected that the tinnitus percept covers the entire normal audible frequency range, and would be a broadband percept. The fact that some subjects reported a tonal tinnitus is clearly inconsistent with this expectation. Moreover, if the bandwidth of tinnitus changed due to gaze, it was always a reduction. Apparently, in complete hearing loss, lateral gaze may enhance spontaneous activity or synchronicity in certain frequency channels relative to neighboring channels.

\section{Cortical activity due to gaze}

Our fMRI experiment showed that peripheral gaze resulted in both activated and deactivated areas in the occipital lobes (Fig. 4). These effects were observed across all subjects. Deactivated areas coincided with the primary visual cortex. This observation is in 
Auditory areas

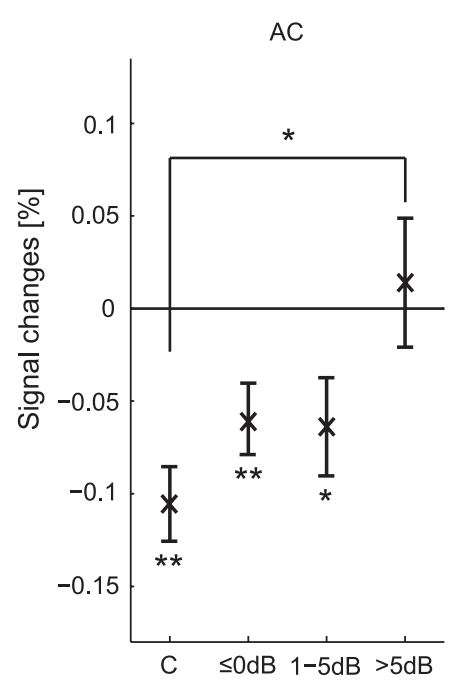

${ }^{*} \quad p<0.05$
${ }^{\star *} \quad p<0.001$

Visual areas
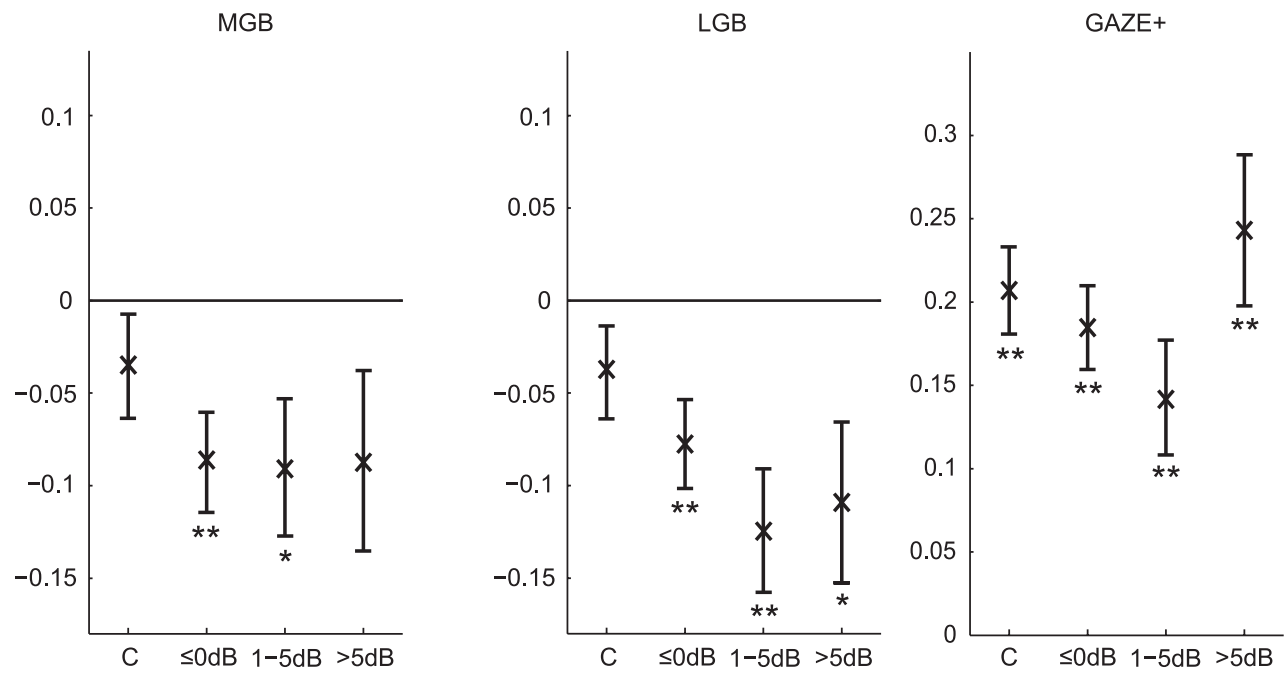

IC
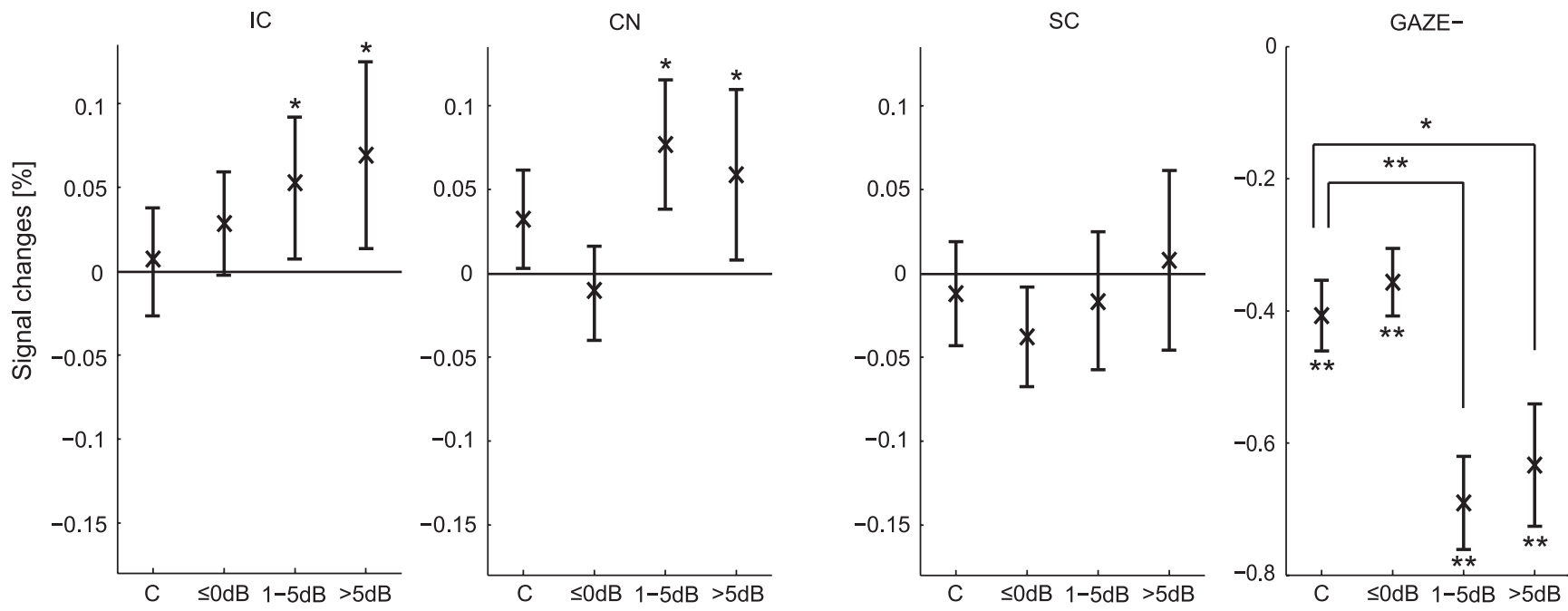

Figure 7. ROI responses to peripheral gaze related to change in tinnitus loudness. Controls and tinnitus subjects are shown separately. Control subjects are copied from Figure 5. Responses are stratified with respect to the perceived amount of intensity increase of the modulated tinnitus ( $\leq 0 \mathrm{~dB}, 1-5 \mathrm{~dB}$, and $>5 \mathrm{~dB}$ ). In total, 49, 19 , and 22 responses were included in the sets $\leq 0 \mathrm{~dB}, 1-5$ $\mathrm{dB}$, and $>5 \mathrm{~dB}$, respectively. The number of right/left surgery subjects for each mentioned set equaled $7 / 7,5 / 2$, and $5 / 3$ subjects, respectively. Error bars indicate the SEs around the mean. Statistical significances against baseline and differences between the sets and the control group are represented by asterisks.

line with the results from an fMRI study performed by Andersson et al. (2007). They observed that the activity in the primary visual cortex was modulated by the position of the eyes with a maximum response occurring when both eyes and head orientations were centrally aligned. In other words, deviation from the central axis with the eyes yielded a relative deactivation in the primary visual cortex, which is consistent with our results.

Alternatively, one may explain the deactivation by eye movements to result from a difference in visual input to the eyes. The subjects saw a cross on a screen when looking straight ahead. When the cross was replaced by an arrow, the subject gazed in the direction indicated by the arrow, toward an essentially dark part in the scanner bore. This darkening of the central visual field may account for the deactivation in the primary visual cortex.
In contrast to the deactivation of the primary visual areas, the precuneus activated with peripheral gaze. This brain region is involved in spatial attention (Culham et al., 1998; Le et al., 1998; Simon et al., 2002). The instruction to gaze in various directions may have shifted and enhanced the subject's visuospatial attention, resulting in increased activity.

In the control subjects, peripheral gaze caused extensive inhibition of the auditory cortices. This can be interpreted to be a manifestation of cross-modal inhibition, as has been observed in several other studies. For example, engagement in a visual navigation task results in extensive inhibition of the auditory cortices (Drzezga et al., 2005). Similarly, a visual detection task inhibits the AC (Mozolic et al., 2008). The latter study also showed that processing of visual information is not required per se but that simply directing attention to the visual modality without present- 
ing a target is sufficient to observe the attentional modulation. The present study supports that peripheral gazing alone is sufficient to inhibit auditory areas.

Cross-modal mechanisms and brainstem activity due to gaze GET typically develops after surgical removal of a vestibular schwannoma in the cerebellopontine angle. In most cases, the surgical removal of the tumor leads to total deafness on the side of the surgery due to transection of cranial nerve VIII and hence sudden complete deafferentation of the $\mathrm{CN}$ in the brainstem (Wiegand and Fickel, 1989; Kane et al., 1995; Bateman et al., 2000). Obviously, the effect of gaze on tinnitus must be an abnormal cross-modal interaction. It has been suggested that deafferentation is a key factor that initiates plastic reorganization, which leads to the abnormal percept (Salvi et al., 2000). The sudden nature of the deafferentation may also account for the reports of GET in other cases of sudden unilateral deafness (Biggs and Ramsden, 2002).

The modulating effect of peripheral gaze on tinnitus has been proposed to originate from reorganization of the functional connections in the lower brainstem. Whittaker $(1982,1983)$ forwarded that neuronal sprouting from the para-abducens nucleus, abducens nucleus, or median longitudinal fasciculus to the $\mathrm{CN}$ is responsible for the development of GET. Alternatively, somatosensory input may be involved in the generation of GET. The trigeminal nerve that innervates the face projects to auditory brainstem areas (Shore et al., 2007), which may provide a pathway that mediates the cross-modal interaction. Both these models would predict that peripheral gaze results in increased activity in the auditory brainstem. In line with this prediction, Lockwood et al. (2001) found increased activity of the dorsal CN. In our study, increased activity could be detected in the $\mathrm{CN}$ as well as in the IC. Thus, the results of Lockwood et al. (2001) and those presented in the current paper are consistent with models that explain GET on the basis of cross-modal interactions in the brainstem.

\section{Activity across the auditory pathway and tinnitus mechanisms}

Our study was initially intended to elucidate the mechanisms of GET specifically. However, from the relations that we observed between the GET loudness and brain activity across the auditory pathway, we may try to draw conclusions on models of tinnitus in general as well.

Notably, we did not observe a relation between the lateralization of tinnitus and that of brain activation. It has repeatedly been shown that monaurally perceived sound results in a response lateralized to the contralateral hemisphere (Suzuki et al., 2002; Langers et al., 2005; this work, Fig. 3a-b). However, although the tinnitus subjects described their tinnitus to be lateralized toward the deaf ear, a corresponding contralateral lateralization was not present in the brain responses (Fig. 6). Apparently, sounds from external acoustic sources and internally generated tinnitus clearly differ from each other in their representation in the brain.

Arguably the most remarkable finding of our study is that an increase of the tinnitus loudness, accompanied by increased $\mathrm{CN}$ and IC activity, did not correspond to an increase of the activity in the MGB. Instead, peripheral gaze decreased MGB activity in the tinnitus subjects in a manner unrelated to loudness. If this decrease is induced by activity in the IC, it must be assumed that the functional connections from the midbrain to the MGB are dominantly inhibitory. This would suggest that the sudden deafferentation due to the tumor surgery reduced the excitatory connections from the IC to the MGB, while the inhibitory connections remained intact.

The decrease of activity in the MGB and the reduced inhibition in the AC has been suggested to be a manifestation of a dysregulation of activity in thalamocortical loops (Llinás et al., 1999). Changes in this loop have been proposed by Llinás and et al., (1999) to account for the observation that tinnitus is associated with increased theta and decreased alpha activity of the brain (Weisz et al., 2011). They proposed that prolonged hyperpolarization of cells in the thalamus produces low-threshold spike bursts and locks the related thalamocortical loops in lowfrequency theta resonance. These low-frequency circuits interact with high-frequency loops at the cortical level, resulting in the generation of a symptom (i.e., tinnitus). The low-frequency hypometabolic areas may be revealed by a reduced blood supply to the MGB, and hence a decreased BOLD response. In the cortex, the dysregulation of the thalamocortical loop leads to hyperactivity, and hence an increased BOLD response. A decrease in the thalamus and an increase in the cortex has already been found in a study of neuralgic pain (Hsieh et al., 1995). In GET patients, Lockwood et al. (2001) previously reported decreased inhibition in the AC. Our study is the first to show hypoactivity and hyperactivity in the thalamus and cortex, respectively, that is associated with tinnitus, as predicted by Llinás et al. (1999).

\section{Conclusion}

GET was found to be associated with reduced inhibition of the auditory cortex, increased activity of the $\mathrm{CN}$ and IC, and inhibition of activity in the MGB. The increased colliculus activity is consistent with models that explain the modulation effect in terms of reorganization of the abducens nucleus or trigeminal projections to auditory brainstem structures. The inhibition of the MGB supports a general tinnitus model that explains tinnitus as dysrhythmia of thalamocortical loops, resulting in slow-wave hypometabolic activation of the thalamus.

\section{References}

Andersson F, Joliot M, Perchey G, Petit L (2007) Eye position-dependent activity in the primary visual area as revealed by fMRI. Hum Brain Mapp 28:673-680. CrossRef Medline

Baguley DM, Phillips J, Humphriss RL, Jones S, Axon PR, Moffat DA (2006) The prevalence and onset of gaze modulation of tinnitus and increased sensitivity to noise after translabyrinthine vestibular schwannoma excision. Otol Neurotol 27:220-224. CrossRef Medline

Bateman N, Nikolopoulos TP, Robinson K, O’Donoghue GM (2000) Impairments, disabilities, and handicaps after acoustic neuroma surgery. Clin Otolaryngol Allied Sci 25:62-65. CrossRef Medline

Baumgart F, Kaulisch T, Tempelmann C, Gaschler-Markefski B, Tegeler C, Schindler F, Stiller D, Scheich H (1998) Electrodynamic headphones and woofers for application in magnetic resonance imaging scanners. Med Phys 25:2068-2070. CrossRef Medline

Biggs ND, Ramsden RT (2002) Gaze-evoked tinnitus following acoustic neuroma resection: a de-afferentation plasticity phenomenon? Clin Otolaryngol Allied Sci 27:338-343. CrossRef Medline

Cacace AT, Lovely TJ, Winter DF, Parnes SM, McFarland DJ (1994) Auditory perceptual and visual-spatial characteristics of gaze-evoked tinnitus. Audiology 33:291-303. CrossRef Medline

Cacace AT, Cousins JC, Moonen CTW, van Gelderen P, Miller D, Parnes SM, Lovely TJ (1995) In-vivo localization of phantom auditory perceptions during functional magnetic resonance imaging of the human brain. In: Proceedings of the fifth international tinnitus seminar (Reich GE, Vernon JE, eds), pp 397-401. Portland, OR: American Tinnitus Association.

Culham JC, Brandt SA, Cavanagh P, Kanwisher NG, Dale AM, Tootell RB (1998) Cortical fMRI activation produced by attentive tracking of moving targets. J Neurophysiol 80:2657-2670. Medline

Drzezga A, Grimmer T, Peller M, Wermke M, Siebner H, Rauschecker JP, 
Schwaiger M, Kurz A (2005) Impaired cross-modal inhibition in Alzheimer disease. PLoS Med 2:e288. CrossRef Medline

Eggermont JJ (2000) Psychological mechanisms and neural models. In: Tinnitus handbook (Tyler RS, ed), pp 85-122. San Diego: Singular Thomson Learning.

Giraud AL, Chéry-Croze S, Fischer G, Fischer C, Vighetto A, Grégoire MC, Lavenne F, Collet L (1999) A selective imaging of tinnitus. Neuroreport 10:1-5. CrossRef Medline

Good P (2002) Extensions of the concept of exchangeability and their applications. J Mod Appl Stat Methods 1:243-247.

Hall DA, Haggard MP, Akeroyd MA, Palmer AR, Summerfield AQ, Elliott MR, Gurney EM, Bowtell RW (1999) "Sparse" temporal sampling in auditory fMRI. Hum Brain Mapp 7:213-223. CrossRef Medline

Hawley ML, Melcher JR, Fullerton BC (2005) Effects of sound bandwidth on fMRI activation in human auditory brainstem nuclei. Hear Res 204: 101-110. CrossRef Medline

Hsieh JC, Belfrage M, Stone-Elander S, Hansson P, Ingvar M (1995) Central representation of chronic ongoing neuropathic pain studied by positron emission tomography. Pain 63:225-236. CrossRef Medline

Kaltenbach JA (2011) Tinnitus: models and mechanisms. Hear Res 276:52_ 60. CrossRef Medline

Kane NM, Kazanas S, Maw AR, Coakham HB, Torrens MJ, Morgan MH, Stranjalis G, Butler SR (1995) Functional outcome in patients after excision of extracanalicular acoustic neuromas using the suboccipital approach. Ann R Coll Surg Engl 77:210-216. Medline

König O, Schaette R, Kempter R, Gross M (2006) Course of hearing loss and occurrence of tinnitus. Hear Res 221:59-64. CrossRef Medline

Langers DR, van Dijk P, Backes WH (2005) Lateralization, connectivity and plasticity in the human central auditory system. Neuroimage 28:490499. CrossRef Medline

Langers DR, de Kleine E, van Dijk P (2012) Tinnitus does not require macroscopic tonotopic map reorganization. Front Syst Neurosci 6:2. Medline

Langguth B, et al. (2007) Consensus for tinnitus patient assessment and treatment outcome measurement: tinnitus Research Initiative meeting, Regensburg, July 2006. Prog Brain Res 166:525-536. CrossRef Medline

Le TH, Pardo JV, Hu X (1998) 4 T-fMRI study of nonspatial shifting of selective attention: cerebellar and parietal contributions. J Neurophysiol 79:1535-1548. Medline

Levine RA (1999) Somatic (craniocervical) tinnitus and the dorsal cochlear nucleus hypothesis. Am J Otolaryngol 20:351-362. CrossRef Medline

Levine RA, Nam EC, Oron Y, Melcher JR (2007) Evidence for a tinnitus subgroup responsive to somatosensory based treatment modalities. Prog Brain Res 166:195-207. CrossRef Medline

Liu RY (1988) Bootstrap procedures under some non-I.I.D. models. Ann Statist 16:1696-1708. CrossRef

Llinás RR, Ribary U, Jeanmonod D, Kronberg E, Mitra PP (1999) Thalamocortical dysrhythmia: a neurological and neuropsychiatric syndrome characterized by magnetoencephalography. Proc Natl Acad Sci U S A 96: 15222-15227. CrossRef Medline

Lockwood AH, Wack DS, Burkard RF, Coad ML, Reyes SA, Arnold SA, Salvi RJ (2001) The functional anatomy of gaze-evoked tinnitus and sustained lateral gaze. Neurology 56:472-480. CrossRef Medline
Lockwood AH, Salvi RJ, Burkard RF (2002) Tinnitus. N Eng J Med 347: 904-910. CrossRef Medline

Maldjian JA, Laurienti PJ, Kraft RA, Burdette JH (2003) An automated method for neuroanatomic and cytoarchitectonic atlas-based interrogation of fMRI data sets. Neuroimage 19:1233-1239. CrossRef Medline

Moore BC, Vinay, Sandhya (2010) The relationship between tinnitus pitch and the edge frequency of the audiogram in individuals with hearing impairment and tonal tinnitus. Hear Res 261:51-56. CrossRef Medline

Mozolic JL, Joyner D, Hugenschmidt CE, Peiffer AM, Kraft RA, Maldjian JA, Laurienti PJ (2008) Cross-modal deactivations during modalityspecific selective attention. BMC Neurol 8:35. CrossRef Medline

Nichols TE, Holmes AP (2002) Nonparametric permutation tests for functional neuroimaging: a primer with examples. Hum Brain Mapp 15:1-25. CrossRef Medline

Norena A, Micheyl C, Chéry-Croze S, Collet L (2002) Psychoacoustic characterization of the tinnitus spectrum: implications for the underlying mechanisms of tinnitus. Audiol Neurootol 7:358-369. CrossRef Medline

Roberts LE, Eggermont JJ, Caspary DM, Shore SE, Melcher JR, Kaltenbach JA (2010) Ringing ears: the neuroscience of tinnitus. J Neurosci 30: 14972-14979. CrossRef Medline

Salvi RJ, Lockwood A, Burkard R (2000) Neural plasticity and tinnitus. In: Tinnitus handbook (Tyler RS, ed) pp 123-148. San Diego: Singular Thomson Learning.

Sereda M, Hall DA, Bosnyak DJ, Edmondson-Jones M, Roberts LE, Adjamian P, Palmer AR (2011) Re-examining the relationship between audiometric profile and tinnitus pitch. Int J Audiol 50:303-312. CrossRef Medline

Shore S, Zhou J, Koehler S (2007) Neural mechanisms underlying somatic tinnitus. Prog Brain Res 166:107-123. CrossRef Medline

Simmons R, Dambra C, Lobarinas E, Stocking C, Salvi R (2008) Head, neck, and eye movements that modulate tinnitus. Semin Hear 29:361-370. CrossRef Medline

Simon SR, Meunier M, Piettre L, Berardi AM, Segebarth CM, Boussaoud D (2002) Spatial attention and memory versus motor preparation: premotor cortex involvement as revealed by fMRI. J Neurophysiol 88: 2047-2057. Medline

Suzuki M, Kitano H, Kitanishi T, Itou R, Shiino A, Nishida Y, Yazawa Y, Ogawa F, Kitajima K (2002) Cortical and subcortical activation with monaural monosyllabic stimulation by functional MRI. Hear Res 163:3745. CrossRef Medline

Weisz N, Hartmann T, Müller N, Lorenz I, Obleser J (2011) Alpha rhythms in audition: cognitive and clinical perspectives. Front Psychol 2:73. Medline

Whittaker CK (1982) Tinnitus and eye movement. Am J Otol 4:188. Medline

Whittaker CK (1983) Intriguing change in tinnitus with eye movement. Am J Otol 4:273. CrossRef Medline

Wiegand DA, Fickel V (1989) Acoustic neuroma-the patient's perspective: subjective assessment of symptoms, diagnosis, therapy, and outcome in 541 patients. Laryngoscope 99:179-187. Medline

Wu CFJ (1986) Jackknife, bootstrap and other resampling methods in regression analysis. Ann Statist 14:1261-1295. CrossRef 